\title{
Raúl Prebisch y la dinámica económica: crecimiento cíclico e interacción entre el centro y la periferia
}

\author{
Esteban Pérez Caldentey y Matias Vernengo
}

RESUMEN

Prebisch pensaba que para comprender la evolución de las economías capitalistas en el tiempo y en diferentes contextos se requería una teoría general del ciclo que abarcara las diferentes áreas de la actividad económica, a la que denominó "dinámica económica". Esta teoría, elaborada entre 1945 y 1949, surgió como crítica de las teorías neoclásica y keynestiana, que Prebisch consideraba representaciones estáticas del capitalismo. Se aplicó primero a una economía cerrada y luego a un contexto de centro-periferia. En ella se combinaba la noción de que los beneficios son la fuerza motriz de la actividad económica, con un proceso de ahorro forzado y la idea de que el desfase temporal entre la circulación de ingresos (y la consiguiente demanda) y la finalización del proceso productivo constituyen la principal fuente de fluctuaciones cíclicas. La teoría dinámica de Prebisch, que nunca llegó a completar, influyó en la elaboración del "Manifiesto" (Prebisch, 1949). 


\section{I}

\section{Introducción}

Raúl Prebisch (1901-1986) sostenía que el ciclo es la manera natural en que las economías capitalistas evolucionan y crecen con el paso del tiempo. Afirmaba también que el ciclo asume una forma particular, ondulatoria, que caracteriza a todos los aspectos de la actividad económica, incluidos la producción, el empleo y la distribución. Después de dejar el Banco Central de la República Argentina en 1943, Prebisch comenzó a trabajar en el desarrollo de una teoría general del ciclo que pudiera explicar el movimiento ondulatorio en las economías capitalistas, a la que daría el nombre de "dinámica económica".

Prebisch desarrolló sus ideas en dos etapas. En la primera, de 1943 a 1948, se concentró en la crítica de la teoría económica vigente. A su entender, los análisis clásico y keynesiano no lograron comprender e incorporar las características cíclicas esenciales del capitalismo y, por ese motivo, distaban de la realidad de funcionamiento de las economías de libre mercado. En la economía clásica se examinaba el capitalismo en un marco estático e intemporal, limitado por la tendencia a la plena utilización de los recursos mediante variaciones de los precios relativos y, en particular, de la tasa de interés.

De acuerdo con Prebisch, la economía de Keynes presentaba el mismo defecto. A pesar de sus declaraciones en contrario, Keynes nunca pudo escapar de los hábitos mentales del pensamiento clásico. Su análisis, en particular la Teoría general de la ocupación, el interés y el dinero (1943), también se mantuvo anclado en una concepción intemporal del capitalismo. Keynes sustituyó el ajuste de los precios relativos de la economía clásica, y específicamente el ajuste de las tasas de interés, por un enfoque basado en las variaciones de los ingresos (el

Las opiniones expresadas en este documento son de exclusiva responsabilidad de los autores y no necesariamente coinciden con las de las instituciones a las que pertenecen. Se agradece a Robert Blecker, Mario Cimoli, Gabriel Porcile, Wilson Peres, Mark Setterfield y a los estudiantes del curso de 2011 de la Escuela de Verano sobre Economías Latinoamericanas de la CEPAL por sus valiosos comentarios acerca de una versión anterior de este trabajo, presentada en el seminario internacional de la CEPAL sobre Structural Dynamics, Growth, Innovation and Distribution (agosto de 2011), y a Ivo Maes y otros participantes de la conferencia de la European Society for the History of Economic Thought (ESHET): "Países de centro y periferia: lecciones de la historia económica y de la historia del pensamiento económico", realizada en Buenos Aires en noviembre de 2012. multiplicador), que supuso la equiparación inmediata de inversión y ahorro.

Prebisch articuló parcialmente esas opiniones críticas en una serie de conferencias dictadas en Buenos Aires entre abril de 1945 y octubre de 1948 con el título de Economía política (dinámica económica). En las primeras conferencias realizó una crítica de la economía clásica y de la Teoría General de Keynes, bajo el título "La crisis de la Economía Política. Keynes. Los clásicos"1. Como parte del desarrollo de su teoría dinámica, el análisis de la obra de Keynes llevó a Prebisch a publicar su Introducción a Keynes, donde explicaba las principales ideas de la Teoría General (Prebisch, 1947a).

En una segunda etapa, desde 1948 hasta su incorporación a la Secretaría Ejecutiva de la CEPAL en 1949, Prebisch comenzó a sentar las bases de un enfoque alternativo que explicara el movimiento ondulatorio en las economías capitalistas, captando precisamente el elemento que faltaba en los análisis clásico y keynesiano. La mayoría de las ideas que llevaron a Prebisch a elaborar su modelo alternativo se desarrollaron durante una serie de conferencias dictadas en la Universidad de Buenos Aires en 1948 y en la Escuela Nacional de Economía de Ciudad de México en febrero y marzo de $1949^{2}$. Las conferencias de Buenos Aires se titularon "Dinámica económica" y las de Ciudad de México "Teoría dinámica de la economía (con especial aplicación a las economías latinoamericanas)".

El modelo dinámico de Prebisch consiste en una economía con dos clases sociales (trabajadores y empresarios) y dos esferas de actividad (producción y circulación), que produce bienes de consumo final esencialmente con capital circulante. En el modelo

\footnotetext{
${ }^{1}$ En 1947, en el programa del curso de Economía Política (dinámica económica) se incluían 11 secciones separadas. Las primeras cuatro se concentraban en el patrón oro, Bretton Woods y el valor del dinero y la inflación, mientras que en las siete restantes se abordaban las teorías del ciclo y su verificación empírica, haciendo hincapié en el caso de la Argentina (incluidas las políticas aplicadas por el Banco Central bajo el mandato de Prebisch). Véase Prebisch (1947b).

2 La primera conferencia en México se dictó el 18 de febrero de 1949 y la última el 1 de marzo de 1949 (Prebisch, 1993a, vol. 4, págs. 410489). Véanse las notas a pie de página de la "Teoría dinámica de la economía (I)" (Prebisch, 1993b) e "Introducción al curso de dinámica económica” (Prebisch, 1948a). Véase también Prebisch (1948c).
} 
también se incluye un sistema bancario que se adapta pasivamente a la demanda de crédito. La introducción de una dimensión temporal confiere al modelo un carácter dinámico.

Esa dimensión interviene en el ámbito de la producción, pues entre el comienzo de un determinado proceso productivo y su finalización debe pasar cierto tiempo. También interviene en el ámbito de la circulación, pues la masa salarial se gasta completamente en los productos terminados destinados al consumo, pero regresa a la clase empresarial en forma de ingresos después de un período. Prebisch sostenía que el proceso de producción es más largo que el de circulación y que es precisamente esa desigualdad en la duración de ambos procesos la que genera el movimiento ondulatorio en una economía capitalista, es decir, su dinámica.

Mediante el análisis dinámico, Prebisch quería demostrar que, si el movimiento ondulatorio en las economías capitalistas se entendiera correctamente, las fuerzas económicas nunca conducirían a un punto de equilibrio, sino a una serie de períodos de expansión y recesión en cuyo marco crece la economía. Esta era su manera de entender el crecimiento cíclico. Con el tiempo amplió su análisis dinámico para incluir la relación entre el centro y la periferia.

En marzo de 1949, al final de sus conferencias sobre dinámica económica, Prebisch estaba convencido de que su teoría tenía carácter general y no se limitaba a un determinado contexto o a circunstancias específicas. Sin embargo, debido a problemas de tiempo y a compromisos urgentes, nunca desarrolló completamente su teoría dinámica. En 1949 comenzó a trabajar para la entonces llamada Comisión Económica para América Latina (CEPAL), primero en calidad de consultor y dos años más tarde, como Secretario Ejecutivo. Para entonces, su interés se había orientado al problema de la industrialización en América Latina, como demuestra la publicación de El desarrollo económico de la América Latina y algunos de sus principales problemas (Prebisch, 1949), de su Manifiesto, y de "Crecimiento, desequilibrio y disparidades: interpretación del proceso de desarrollo económico" (Prebisch, 1951b), trabajos que sin embargo estaban claramente influenciados por sus análisis del ciclo y de la dinámica económica.

En el presente trabajo se realiza un análisis crítico y sistemático de la dinámica económica de Prebisch. El artículo se divide en ocho secciones, incluida esta Introducción. En la segunda sección se examinan sus críticas a la teoría neoclásica y a la Teoría General de Keynes como representaciones estáticas del desarrollo capitalista, mientras que de la tercera a la sexta secciones se presentan las bases de la teoría dinámica de Prebisch y su desarrollo. En la séptima sección se analiza la manera en que él la aplicó a la relación entre el centro y la periferia y se argumenta que, según el autor, la teoría dinámica era general y no específica, de modo que resultaba posible incorporar diferentes supuestos, parámetros y situaciones variables sin modificar sus fundamentos y análisis centrales. En la octava y última sección se presentan las conclusiones.

\section{II}

\section{La teoría neoclásica y la Teoría General de Keynes: dos representaciones estáticas del capitalismo}

Prebisch pensaba que el proceso de crecimiento en las economías capitalistas era eminentemente cíclico. Como él mismo dijo: "El ciclo es la típica forma de crecimiento que el capitalismo ha tenido históricamente y sigue teniendo. La actividad económica [...] se expande y contrae continuamente en una sucesión interrumpida de las fases de crecimiento en los ingresos, en la ocupación y en la producción, seguida de fases de decrecimiento con la consiguiente declinación de la producción y la ocupación" (Prebisch, 1948a) ${ }^{3}$. Su manera de entender el capitalismo es típica de muchos autores del período. Para Prebisch, la realidad cíclica del crecimiento capitalista socava completamente la noción de equilibrio, entendido en el sentido de Adam Smith como centro de gravedad, y con mayor razón al considerarlo desde el

\footnotetext{
${ }^{3}$ Véase también Prebisch (2003a).
} 
punto de vista de la noción neoclásica de eficiencia en la asignación. Como Prebisch señaló: "No hay punto de reposo: se asciende para descender y se desciende para ascender nuevamente. En ese movimiento no hay punto de equilibrio; ese movimiento es una sucesión continua de desequilibrios" (Prebisch, 1948a). Esta idea se aplica tanto a los equilibrios estáticos como dinámicos.

Prebisch llegó a ser muy crítico de la teoría económica predominante porque esta se anclaba en el análisis del equilibrio y estaba, por lo tanto, divorciada de la realidad capitalista. En su opinión, tanto la teoría neoclásica como la keynesiana presentaban el mismo defecto.

Su crítica a la teoría neoclásica se concentra en la teoría de la productividad marginal. De acuerdo con Prebisch, en esta última se suponía que la evolución de una economía de mercado solo podía caracterizarse por una línea de avance regular y, en términos más modernos, solo era compatible con el crecimiento en el estado estacionario, concepto y expresión que se convirtieron en una importante herramienta de la corriente principal recién una década más tarde. Prebisch entendió, correctamente, que el crecimiento en el estado estacionario está determinado por la tasa de aumento de la población y la innovación técnica ${ }^{4}$. Para él, la noción de crecimiento en el estado estacionario era antitética a la propia naturaleza de la evolución capitalista.

Prebisch ilustra ese punto con un modelo implícito que comprende un sector de bienes de consumo e inversión. La aplicación de la teoría de la productividad marginal a este tipo de modelo significa que si la producción se expande de manera constante, la inversión y el consumo también deberían aumentar a una tasa de crecimiento constante y, por ende, mantener una relación dada en el tiempo. En otras palabras, la teoría de la productividad marginal presupone un proceso de crecimiento equilibrado, de manera que las condiciones competitivas aseguran que la producción de capital mantenga una relación dada con respecto a los bienes de consumo, determinada por la tasa de crecimiento de la población, la innovación técnica y las preferencias. Las divergencias con respecto a esa relación entre la inversión y el consumo se eliminan mediante cambios en los recursos derivados de la diferencia entre el producto marginal del capital y la tasa de interés. Si la razón entre la inversión y los bienes de consumo supera (no alcanza) la razón compatible con el crecimiento equilibrado, el producto marginal del capital estaría por debajo (sobre) de la tasa de interés, causando una

\footnotetext{
4 Véase Prebisch (1948f).
}

transferencia de recursos de la producción de capital (consumo) a los bienes de consumo (inversión). Esto conduciría a un aumento (disminución) del producto marginal del capital hasta alcanzar la igualdad con la tasa de interés. Como explica Prebisch:

"La relación lógica entre industrias de capital e industrias de consumo está dada por el ritmo de crecimiento de la población, el de las innovaciones técnicas y las preferencias de ahorro de la colectividad. Por lo tanto, si por cualquier razón en una industria determinada, se hubiera creado una capacidad superior a la que justifica el consumo, puesto que la inversión es un mero anticipo del consumo que se prevé, si existiera entonces exceso de capacidad, la productividad del incremento de capital, que ha traído consigo ese exceso, estaría por debajo del tipo de interés y eliminaría ese exceso. De manera que no se concibe ni en casos ni sectores particulares de la economía un exceso de capacidad de producción de artículos o bienes de capital (...)" (Prebisch, 1948f).

Desde este punto de vista, la tasa de interés actúa como un centro de gravedad para el producto marginal del capital y es el principal mecanismo de coordinación de la actividad económica. Prebisch consideraba la tasa de interés, en el sentido definido por los autores neoclásicos, como un artificio, no obstante útil, porque permitía a la teoría neoclásica mantenerse dentro de los límites de la teoría del equilibrio intemporal, incluso al analizar una economía de producción capitalista. Para Prebisch, el proceso de producción requiere el paso del tiempo o de un período durante el cual se deben generar ahorros y transformarlos en capital. Como él sostuvo:

“(...) transcurre un tiempo más o menos largo entre la iniciación de las operaciones y la terminación del producto final que ha de ponerse a disposición del consumidor. Mientras tanto, los factores productivos que están ocupados en esa posición intermedia que ha de crear — con el andar del tiempo- el producto final, necesitan consumir y si ellos mismos no ahorran, es decir, si ellos mismos se dejan de consumir - lo cual ocurre sólo en pequeño grado- otros factores dentro de la colectividad deben dejar de consumir para que ellos consuman. Dejar de consumir es ahorrar y transferir lo que dejan de consumir unos a los otros que están trabajando en la producción intermedia. Es invertir ese ahorro en la producción intermedia, es decir, invertir ese ahorro en la formación de capital, puesto que la producción intermedia — todo aquello que está en proceso de transformación en 
productos finales, proceso más o menos largo- es el capital" (Prebisch, 1948b, pág. 272).

La teoría clásica permite que el proceso de transformación del ahorro en capital esté perfectamente sincronizado por los cambios en la tasa de interés. La tasa de interés es el "artificio [artificio, es el término que utiliza Prebisch] teórico", como él lo denominó, de la sincronía necesaria. De acuerdo con su punto de vista, la teoría neoclásica sostiene que "no es posible hacer inversiones sin contar con una cantidad equivalente y simultánea de ahorro" (Prebisch, 1948b). En otras palabras, la tasa de interés regula el ahorro de manera que el acto de ahorrar y el de invertir sean un acontecimiento simultáneo.

Luego de exponer su crítica a la teoría neoclásica, Prebisch se concentra en el análisis de la obra de John Maynard Keynes, quien a su juicio nunca pudo liberarse completamente de las cadenas de la teoría clásica y se mantuvo apegado a la noción neoclásica de equilibrio. Después de despreciar la teoría neoclásica de las tasas de interés, que criticó profusamente en su Teoría General, Keynes utilizó un artificio alternativo como medio para prescindir del tiempo, al afirmar que el multiplicador y los cambios relacionados en los ingresos generarían el ahorro necesario para "financiar" las inversiones. Prebisch interpreta el multiplicador como un "multiplicador instantáneo" y sostiene que:

"El aspecto revolucionario de Keynes, desde un punto de vista teórico, es su famosa teoría del multiplicador, que es otra teoría del equilibrio económico, cuya estructura es muy similar a la vieja teoría cuantitativa del dinero. Tal es la fuerza de ese hábito mental, del que el mismo Keynes no se emanciparía nunca. Fue así que siguió a los economistas clásicos a donde menos tendría que haberlos seguido: en la búsqueda de leyes de equilibrio, que es, en mi opinión, el mayor obstáculo al progreso de la economía política desde un punto de vista teórico. Si estos obstáculos no se eliminan enérgicamente, continuaremos formulando constructos desconectados de la realidad" (Prebisch, 1948a).

Su explicación continúa de la siguiente manera:

"O sea que dado un incremento de inversiones, los ingresos crecerán en la medida necesaria para producir en la colectividad un incremento de ahorro equivalente al incremento de las inversiones. Es claro que este razonamiento puede hacerlo un teórico que prescinde del tiempo. ¿Por qué? Porque es indispensable el tiempo para que el incremento de inversiones dé el incremento de ingresos, o sea que en el fondo el pensamiento keynesiano significa decir: para hacer inversiones hoy, usaremos como ahorro el que se haga en el futuro (...) Este razonamiento solamente puede hacerse si se confunde el futuro con el presente eliminando completamente el factor tiempo en el proceso. Tal es una de las grandes inconsistencias lógicas que invalidan la teoría keynesiana"5 (Prebisch, 1948b, pág. 277).

${ }^{5}$ Schumpeter (1946) formuló una crítica similar de la Teoría General de Keynes.

\section{III}

\section{Principales elementos de la teoría dinámica de Prebisch}

\begin{abstract}
Después de afirmar que los constructos intemporales de los autores neoclásicos y de Keynes no son relevantes para comprender el capitalismo, Prebisch hace hincapié en la necesidad de formular una teoría dinámica que capte la dimensión temporal y el movimiento ondulatorio del capitalismo, en el entendido de que dicha teoría abarcaría todo el espectro de la actividad económica. En sus palabras:
\end{abstract}

"Cada vez me convenzo más de que el ciclo es la forma de crecer de la economía capitalista. La economía capitalista no ha crecido sino en una forma ondulatoria, no se ha movido sino en esa forma y cualquier perturbación de conjunto da al movimiento una forma ondulatoria. Por lo tanto, si el ciclo es la forma de crecer y de moverse de la economía y si la economía se mueve incesantemente 
en esa forma, parecería que todos los fenómenos de conjunto de la economía, no sólo los de ocupación y producción sino también los de la distribución, debieran integrarse dentro de una teoría dinámica general" (Prebisch, 1993b, pág. 414).

Una teoría dinámica no solo debería explicar la razón de las fases alternas de prosperidad y depresión que caracterizan a las economías capitalistas, sino también los procesos de producción y distribución ${ }^{6}$.

El análisis dinámico de Prebisch se presenta como un modelo que comprende dos clases sociales, empresarios y trabajadores, y dos esferas de actividad: producción y circulación. Asimismo, asume que el sistema bancario reacciona pasivamente a la demanda de crédito, que la economía produce bienes de consumo final y que los salarios de los trabajadores se gastan completamente en el consumo final, de manera que las empresas recuperan el dinero gastado en la producción.

La variable tiempo se incorpora de manera fundamental en la producción y la circulación. Un proceso de producción no tiene lugar en forma instantánea, sino que requiere tiempo. Entre el comienzo de cualquier proceso productivo y su resultado final (la producción

\footnotetext{
${ }^{6}$ Como señala Prebisch, la teoría de la dinámica económica pretende explicar esta manera [cíclica] en que se producen los fenómenos e identificar sus uniformidades para formular los principios y las leyes que gobiernan ese movimiento. Asimismo, él afirma que la teoría dinámica también desempeña un papel fundamental en el análisis de tres de las principales características de una economía de libre mercado: la inestabilidad del sistema económico, la desigualdad en la distribución del ingreso y el persistente desempleo. En ese sentido, Prebisch comparte algunas de las opiniones de Keynes sobre la inversión y las fallas del capitalismo, a saber "su incapacidad para procurar la ocupación plena y su arbitraria y desigual distribución de la riqueza y los ingresos" (Keynes, 1943). Véase Prebisch (1948b, 1948e, 1948f y 1947a). Prebisch agrega a esa lista la inestabilidad (Prebisch, 1948b y 1948e).
}

de bienes terminados), se agrega un valor al proceso en cada intervalo, de manera que todo el proceso de producción consiste en una suma de valores agregados. Junto con el proceso de creación de valor agregado (proceso de producción) hay un proceso de generación de ingresos. Deben comprarse medios de producción y los participantes del proceso productivo reciben ingresos por su aporte.

Algunos salarios y beneficios se distribuyen antes de que el proceso productivo llegue a su fin. La diferencia de tiempo entre el período en que los ingresos se distribuyen (esfera de la circulación) y el período necesario para completar el proceso de producción (esfera de la producción) crea una disparidad entre la demanda y la oferta agregadas y es la fuente de los beneficios y del ciclo. En otras palabras, el hecho de que las esferas monetaria y real funcionen en diferentes períodos explica por qué las economías evolucionan a través de una serie de posiciones de desequilibrio en lugar de tender a una posición de equilibrio (Prebisch, 1948a).

La fase ascendente del ciclo se caracteriza por un exceso de ingresos con respecto al valor agregado, es decir, un exceso de demanda agregada con relación a la oferta agregada. Esto a su vez se traduce en mayores gastos, precios, beneficios e inversiones. El flujo de gastos por concepto de inversiones regresa con el tiempo al empresario en forma de beneficios.

En la fase descendente del ciclo, a medida que la oferta agregada supera a la demanda agregada, los precios $\mathrm{y}$ los beneficios disminuyen, conduciendo a un proceso de desinversión. Debido a que las fases ascendentes y descendentes se alternan, Prebisch sostenía que "así la economía capitalista se caracteriza por un continuo proceso de inversión y desinversión, con esta particularidad: que por lo general en el proceso de desinversión no se desinvierte todo lo invertido; de lo contrario no habría crecimiento económico" (Prebisch, 1993b, pág. 425).

\section{IV}

\section{Representación esquemática del análisis de Prebisch}

Prebisch amplía su análisis de la dinámica mediante una representación gráfica que consiste en un plano dividido en dos triángulos rectángulos con áreas iguales. El primer triángulo $(\mathrm{ADF})$ representa la producción en proceso.
El segundo triángulo (FDK) representa la producción terminada. En esta etapa, Prebisch tiene en cuenta solo el capital circulante. El objetivo del plano de dos triángulos consiste en captar las tres dimensiones del proceso de 
producción: el tiempo transcurrido durante este proceso; la producción real, incluidas la que se halla en proceso y la final (masa de producción); y los ingresos monetarios pagados a los factores que intervienen en el proceso productivo. Prebisch asume que hay nueve procesos de producción y que cada uno requiere de nueve meses para producir una unidad final.

Asume, además, que los salarios solo se pagan mientras la producción está en proceso, que dichos salarios se gastan en productos terminados destinados al consumo final y que no hay beneficios en esta etapa. Los empresarios recuperan los ingresos que pagan a los trabajadores (masa salarial) mediante la venta de productos finales. Prebisch también asume que los ingresos comerciales se reinvierten, a su vez, en el proceso productivo.

La base del triángulo ADF (AD) representa las etapas del proceso de producción, que Prebisch divide en nueve períodos iguales (o meses). La hipotenusa del triángulo $(\mathrm{AF})$ representa tanto los ingresos pagados por la producción en proceso como el comienzo secuencial de cada una de las series (o nueve etapas) de los nueve procesos productivos. La hipotenusa del triángulo FDK (DK) representa los ingresos pagados por los bienes finales (véase el gráfico 1).

Al final de los primeros nueve meses, el primer proceso productivo (indicado por $\mathrm{ABCD}$ en el primer triángulo (AFD)) genera una unidad de bienes finales (indicada por $\mathrm{CDH}$ en el segundo triángulo (FDK)), que es equivalente a ABK. Estos bienes se venden y las ganancias se reinvierten y agregan a la producción en proceso (ADF). En este punto, las empresas comienzan el segundo proceso productivo, que tomará nueve meses. Una vez completado este, la producción final aparece como ICHJ en el segundo triángulo (FDK). ICHJ equivale a la suma de las ganancias reinvertidas (CDH) y el agregado del producto final que deriva del segundo proceso productivo, pero sin tener en cuenta las ganancias reinvertidas de dicha producción (ICHK). De ahí en adelante, el proceso se repite hasta completar nueve procesos productivos, y cada uno contribuye con el mismo volumen en proceso a la producción final. Al finalizar todo el proceso, el ingreso total pagado será igual al ingreso total recibido por la producción final $(\mathrm{AF}=\mathrm{DK}$ o $\alpha=\beta)$.

Este sistema de estado estacionario (que comprende a los trabajadores que reciben salarios que luego se pagan a las empresas por la compra de productos finales y a las empresas que luego reinvierten esas ganancias) solo puede expandirse si los ingresos pagados por la producción en proceso superan los ingresos recibidos por las empresas por la venta del producto final. Esto se muestra en el desplazamiento de AF a AL, que conduce a un aumento de los ingresos pagados a los factores por la producción en proceso que excede a la producción final (DK) $\left(\alpha=\beta\right.$ y $\left.\alpha_{1}>\beta\right)$.

GRÁFICO 1

\section{La dinámica de Prebisch como proceso de capital circulante}

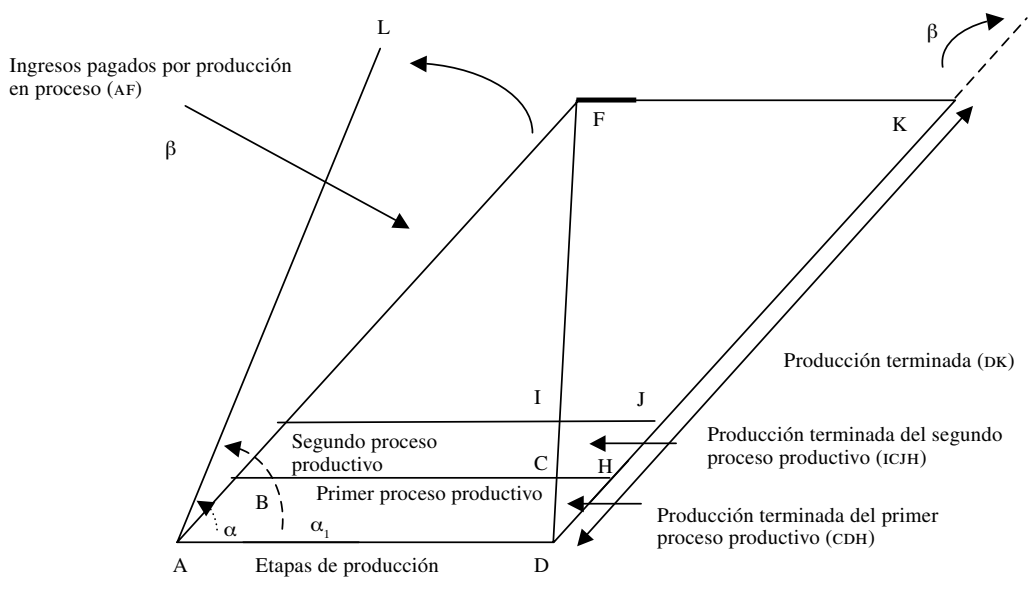

Fuente: Elaboración propia sobre la base de R. Prebisch, "Teoría dinámica de la economía (II)", Raúl Prebisch: Obras, 1919-1948, vol. 4, Buenos Aires, Fundación Raúl Prebisch, 1993. 


\section{V}

\section{Beneficios y ahorro forzado}

En una etapa posterior, Prebisch introduce los beneficios en su análisis. De hecho, estos constituyen el eje sobre el que construye su teoría dinámica. Los beneficios derivan del ahorro forzado.

En sus propias palabras:

“(...) la mayor parte de las inversiones de capital fijo son cubiertas indirectamente con incremento de dinero y no acudiendo al mercado, de acuerdo con las leyes de la oferta y la demanda trazadas en la economía clásica. (...) ¿cómo actúa el incremento de dinero? (...) el incremento de dinero puesto en circulación por las personas para pagar sus inversiones hace subir los precios (...). El alza de precios significa rebaja de salarios reales; (...) para compeler a ahorrar a quienes pagan los mayores precios, trasladando este ahorro al empresario, en forma de artículos de consumo; el empresario lo transfiere a su vez a los ocupados en la formación de capital. (...) la mayor parte del ahorro (...) no es el resultado de un mecanismo de incentivos y preferencias, sino de un mecanismo de compulsión. Se compele a ahorrar en la medida en que no hay suficiente ahorro espontáneo" (Prebisch, 1948c).

Prebisch cree firmemente que el ahorro precede a la inversión, y que la realización de inversiones necesariamente requiere un acto previo de ahorro. Como él señala (Prebisch, 1948c): "Estoy convencido, que el dejar de consumir es esencial para capitalizar inversiones. En esto estoy de acuerdo con las razones que expone la escuela clásica y discrepo con Keynes".

Sin embargo, Prebisch considera que la noción de ahorro forzado es incompatible con la idea ortodoxa neoclásica del ahorro, que lo concibe como el resultado de preferencias individuales. Lo expresa de la siguiente manera: "Gran parte de las inversiones se han realizado históricamente y se siguen realizando por un mecanismo muy distinto que el de la oferta y demanda de ahorro: y gran parte del ahorro que se invierte no es el resultado de lo que prefiera hacer espontáneamente la colectividad en función de sus inclinaciones y gustos y de la tasa de interés" (Prebisch, 1948b).

De acuerdo con Prebisch, los beneficios derivan de un exceso de demanda con respecto a la oferta y se materializan desde el principio hasta el final del proceso productivo. El mecanismo de transmisión a través de los beneficios comprende todo el espectro de empresas involucradas desde la primera hasta la última etapa del proceso productivo: productores de materias primas, industriales, comerciantes mayoristas y minoristas. Prebisch asume que los precios para los minoristas son fijos, mientras que son flexibles para los mayoristas, los fabricantes y los productores de materias primas. Además, los minoristas aspiran a mantener un determinado nivel normal de existencias.

El aumento de la demanda de productos finales provoca una reducción de las existencias de los minoristas por debajo del nivel normal, que conduce a una mayor demanda de productos de los mayoristas y a precios y beneficios más elevados. En respuesta a los precios y beneficios más altos, los mayoristas expanden sus operaciones y aumenta su demanda de productos de los industriales. El mismo mecanismo conduce a un incremento en la demanda de materias primas por parte de los industriales. El crecimiento de la demanda y los mayores precios y beneficios se transmiten entonces a todo el proceso de producción y distribución. En cada etapa, el acrecentamiento de la demanda, los precios y los beneficios es acompañado de un aumento concomitante de la masa salarial (Prebisch, 1993c y 1993d). Este proceso produce un alza de los salarios y los beneficios, que luego se incorporan a los precios cobrados por todos los tipos de empresas entre sí y a los consumidores finales. De ahí que los productos primarios vendidos a los industriales, los productos al por mayor vendidos a los minoristas y los productos finales vendidos a los consumidores habrán incorporado el nuevo nivel de beneficios, que surgió de un desequilibrio entre la oferta y la demanda agregadas.

Prebisch pensaba que una vez que se incorporaba un nivel superior de beneficios en todo el proceso de producción, esos beneficios no podrían quitarse ni actuar como mecanismo de ajuste ante una contracción de la demanda. Así, del mismo modo en que los beneficios y el adelanto de beneficios actúan como disparador para incrementar la producción y los ingresos, también son una importante fuerza de rigidez en el sistema, que le impide operar de la manera en que los economistas clásicos teorizaron. Como él mismo señala:

“(...) los beneficios que se han ido así acumulando en el proceso productivo son irreversibles, de tal suerte que si las conjeturas que han hecho los empresarios 
acerca de la demanda futura y que han provocado esa cristalización anticipada de beneficios en el proceso productivo, resultan alguna vez negadas por la realidad, el producto que se ofrece al mercado ya tiene incorporados los beneficios en forma tal que no se puede reducir, porque han sido pagados en las distintas etapas... Así los valores de oferta en el proceso productivo adquieren una rigidez tal, en virtud de la cristalización del beneficio, que va a provocar luego la contracción cíclica cuando la demanda, después del punto de conjunción, es insuficiente para absorber la oferta" (Prebisch, 1993c).

De acuerdo con ese razonamiento, el nivel de beneficios por unidad producida por cada categoría de empresas, con excepción de los productores de materias primas, está determinado por el margen de beneficios entre las distintas etapas del proceso productivo, que a su vez depende del grado de competencia (o, como expresa Prebisch (1993d), de "competencia y movilidad").

Por otra parte, el nivel de beneficios está determinado por el tiempo que transcurre entre el momento en que se alcanza el nivel de demanda deseado y el momento en que un producto se lleva al mercado. Para los productores de productos básicos, el nivel de beneficios depende solo de la duración del intervalo entre la compra de un producto y su venta. En el caso de los comerciantes mayoristas y minoristas, el tiempo transcurrido entre la compra y la venta comprende las distintas transacciones que se realizan en la esfera de la circulación del proceso productivo y no en la esfera de la producción en sí, como en el caso de las empresas manufactureras (industriales) y los productores de materias primas.

Específicamente, el nivel de beneficios de los minoristas $\left(\pi_{R T}\right)$ estará determinado por un margen de beneficios que sobrepasa el nivel de beneficios de los mayoristas $\left(\pi_{W T}\right.$ y el tiempo que transcurre entre la compra de los productos a los mayoristas y la venta a los consumidores finales. A su vez, el nivel de beneficios de los mayoristas $\left(\pi_{W T}\right)$ estará determinado por un margen de beneficios superior al nivel de beneficios de los industriales $\left(\pi_{I}\right)$ y por el tiempo transcurrido entre la compra y la venta. Por su parte, el nivel de beneficios de los industriales $\left(\pi_{I}\right)$ estará determinado por un margen de beneficios más alto con respecto al nivel de beneficios de los productores de materias primas y por la duración del proceso de manufactura. En el caso de los productores de materias primas, los beneficios estarán determinados solo por el tiempo transcurrido entre la planificación y la extracción de las materias primas y su venta a las industrias manufactureras. Formalmente:

$$
\begin{gathered}
\pi_{R T}=\mu_{1} \pi_{W T}+\theta_{R T} \\
\pi_{W T}=\mu_{2} \pi_{I}+\theta_{W T} \\
\pi_{I}=\mu_{3} \pi_{R M}+\theta_{I} \\
\pi_{R M}=\theta_{R M}
\end{gathered}
$$

donde $\mu_{i}$ y $\theta_{i}$ representan a los respectivos márgenes de beneficios y duración del proceso productivo en cada categoría de productores o empresas (minoristas y mayoristas, industriales y productores de materias primas). La sucesiva sustitución significa que el nivel de beneficios en el sector minorista depende de los diferentes aumentos de precio aplicados en cada etapa de la producción y la distribución, ponderados por el tiempo transcurrido entre la compra y la venta de materias primas, productos manufacturados y bienes de venta al por mayor y el tiempo necesario para llevar las materias primas al mercado para la venta. Es decir:

$$
\pi_{R T}=\mu_{1}\left[\mu_{2} \mu_{3} \theta_{R M}+\mu_{2} \theta_{I}+\theta_{W T}\right]+\theta_{R T}
$$

En condiciones de competencia perfecta, $\mu_{i}=0 \mathrm{y}$ la existencia de beneficios para los minoristas se explica solo por $\theta_{R T}$. En el caso opuesto, $\mu_{i}=1$, los beneficios derivan del tiempo transcurrido para completar todos los procesos de producción y distribución. Como se aprecia en la ecuación (2), el minorista (dado el supuesto de que realizará las ventas planificadas) obtiene sus propios beneficios y recupera, además, los otros beneficios obtenidos y pagados en cada etapa del proceso productivo. Los beneficios se ganan e incorporan al precio cobrado por cada empresa que interviene en el proceso, incluso antes de que el minorista realice la venta final del producto. Debido a que los minoristas operan con precios fijos, el incremento de la demanda de bienes de consumo conducirá a una reducción de sus existencias. Como se explicó anteriormente, esto provocará un crecimiento de la demanda de productos de venta al por mayor, manufacturas y materias primas por parte, respectivamente, de los minoristas, los mayoristas y las empresas manufactureras. Dado que esas empresas operan en mercados de precios flexibles, el acrecentamiento de la demanda derivará en un aumento de los precios y una expansión de los beneficios en toda la cadena de producción. Los mayores beneficios (y expectativas de beneficios) redundarán en una mayor demanda de inversión, mano de obra y de todos los tipos de bienes utilizados en el proceso, desde la producción de materias primas hasta la venta al por mayor. Esa mayor demanda de mano de obra y bienes se traducirá en un incremento de los salarios y los precios de los artículos. 
Prebisch explica esto de la siguiente manera:

"Ya sabemos que durante las distintas etapas del proceso productivo se han ido anticipando los beneficios. Cuando el minorista adquiere del mayorista los artículos respectivos, le paga todos los beneficios que así se han venido anticipando; y cuando vende estos artículos recupera todos estos beneficios (...) y obtiene además el minorista sus propios beneficios... En otros términos, el consumidor paga al minorista sus beneficios y le devuelve además todos los beneficios que se habían anticipado anteriormente en el proceso productivo de los artículos por comprar. Ahora bien, durante la creciente, en el mismo momento en que el minorista recupera los beneficios que había pagado anteriormente, está pagando beneficios mayores: hay pues un incremento de beneficios. Este incremento dará lugar inmediatamente a un reajuste en todas las etapas pues al pasar la producción en proceso de manos de unos empresarios a otros, recibirá la parte del incremento que le corresponde (...)" (Prebisch, 1993d, pág. 443).

\section{VI}

\section{Aspectos generales de la teoría dinámica de Prebisch}

La visión de conjunto de Prebisch sobre la dinámica puede resumirse con ayuda de uno de los gráficos que figuran en su "Teoría dinámica de la economía (I)" (Prebisch, 1993b) (véase el gráfico 2). En el gráfico se muestran los ámbitos de la demanda y la oferta en el proceso productivo y su interacción en las fases ascendentes y descendentes del ciclo económico. La demanda está determinada por los ingresos obtenidos y gastados. La oferta lo está por el volumen de producción (ya sea producción en proceso o final). En el gráfico 2 se muestran tres curvas parabólicas: los ingresos pagados a los factores de producción por los fabricantes de productos finales; la producción terminada (volumen de productos terminados) y la demanda (D) de productos finales. Las tres curvas suben y evolucionan a diferentes velocidades e ilustran así la estructura temporal de la producción.

En el ámbito de la demanda (ingresos), los ingresos pagados a los medios de producción $\left(I_{p f p}\right)$ dan lugar a la curva de demanda de productos finales (D), que posteriormente se traduce en ingresos que regresan a las empresas $\left(I_{r e}\right)$. En otras palabras, la demanda (D) es equivalente a los ingresos que vuelven a las empresas $\left(I_{r e}\right)$. Durante el proceso productivo, los productores pagan salarios y beneficios por la producción, que en un flujo circular regresan a ellos en forma de ingresos (es decir, beneficios). En el ámbito de la oferta, la producción

GRÁFICO 2

Representación esquemática de las ideas de Prebisch sobre la dinámica, 1949

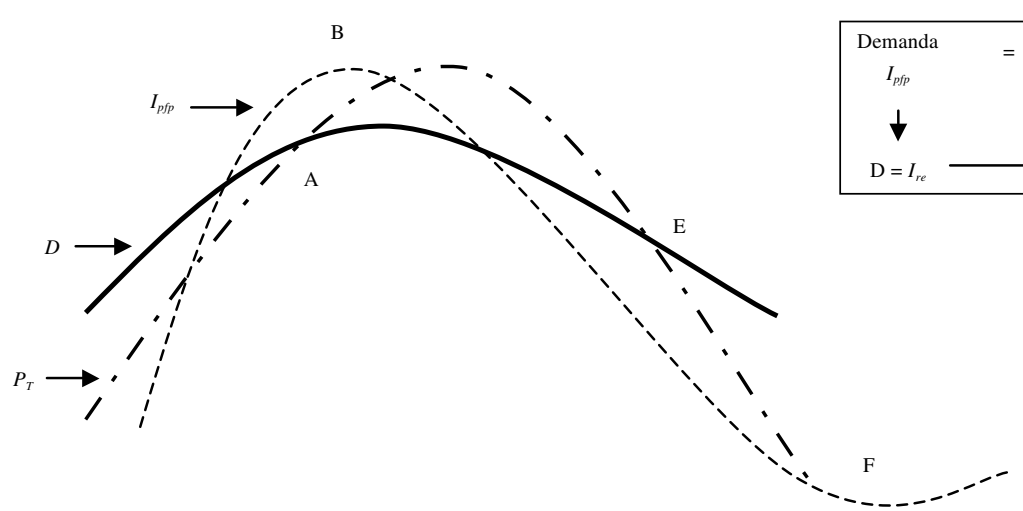

Fuente: Elaboración propia sobre la base de R. Prebisch, "Teoría dinámica de la economía (I)", Raúl Prebisch: Obras, 1919-1948, vol. 4, Buenos Aires, Fundación Raúl Prebisch, 1993. 
en proceso $\left(P_{p}\right)$ da lugar a la curva de producción terminada $\left(P_{T}\right) \mathrm{y}$, por construcción, $P_{p}$ es equivalente a los ingresos pagados a los factores de producción $\left(I_{p f p}\right)$ por los fabricantes de productos finales.

De acuerdo con el gráfico 2, a partir de la fase ascendente del ciclo, los ingresos se pagan a los medios de producción $\left(I_{p f p}\right)$ y la producción en proceso $\left(P_{p}\right)$ tiende inicialmente a dejar atrás a la producción terminada $\left(P_{T}\right)$, debido al retraso en la estructura productiva hasta el punto A. En consecuencia, la demanda (D) durante esta fase es mayor que la producción terminada $u$ oferta. En el punto A, la demanda (D) y la producción terminada $\left(P_{T}\right)$ se intersectan. Sin embargo, de acuerdo con Prebisch, A no es un punto de equilibrio. Esto se debe a que los productores todavía están realizando la producción en proceso $\left(P_{P}\right) \mathrm{y}$, como resultado, incluso en el punto de convergencia de las dos curvas (A), la producción terminada $\left(P_{T}\right)$ aumenta con mayor rapidez que la demanda (D) (es decir, la pendiente de $P_{T}$ en A es más pronunciada que la de $\mathrm{D}$ ). La producción terminada supera a D y continúa ampliándose incluso después de que la demanda ha comenzado a disminuir.

El resultado final es la acumulación de existencias por parte de las empresas y, por consiguiente, una reducción de sus planes de producción en proceso. Esto se aprecia en la disminución de la pendiente de $I_{p f p} \mathrm{y}$, en consecuencia, de $P_{p}$. Su punto de inflexión en B es el comienzo de la fase descendente del ciclo. A medida que $I_{p f p}$ desacelera, $P_{p}$ y D hacen lo mismo.

La disminución de $\mathrm{D}, I_{p f p}$ y $P_{T}$ continúa hasta que la reducción constante de las existencias conduce nuevamente a un punto de convergencia (E) entre la demanda de productos finales (D) y la producción terminada $\left(P_{T}\right)$.
Una vez más, E no es un punto de equilibrio para la demanda (D), que disminuye a un ritmo menor que la producción terminada $\left(P_{T}\right)$. La reducción de las existencias conducirá gradualmente a los empresarios a renovar los pedidos para su proceso de producción, poniendo freno al descenso de $P_{p}$ y $I_{p f p}$ y a la demanda. Eventualmente, $P_{p}$ comenzará a recuperarse (punto $\mathrm{F}$ ) y llevará a $I_{p f p} \mathrm{y}$, en consecuencia, a D hacia una nueva fase ascendente del ciclo $^{7}$.

La representación esquemática de Prebisch muestra, una vez más, que el sello distintivo de la evolución de una economía capitalista no es la tendencia al equilibrio, sino los recurrentes estados de desequilibrio. El desequilibrio es continuo y una situación en que la oferta supera a la demanda conduce a otra en que la demanda supera a la oferta (Prebisch, 1993b). Asimismo, nada garantiza que las fluctuaciones tengan lugar alrededor de un nivel óptimo de utilización de los medios de producción. No obstante la relevancia y originalidad del análisis de la dinámica cíclica de Prebisch, cabe destacar que su aplicación de la dinámica capitalista al análisis de la interacción entre el centro y la periferia fue única.

\footnotetext{
7 En el esquema de Prebisch (Prebisch, 1993b, pág. 419), en la producción final $\left(P_{T}\right)$ y $\left(P_{p}=I_{p f p}\right)$ presentan una pendiente de declive similar, mientras que la pendiente de $\mathrm{D}$ es menos pronunciada. Se estima que esto constituye un error por parte de Prebisch. La reducción de las existencias (que se considera producción en proceso) llevará a los empresarios a ampliar sus pedidos de insumos necesarios para el proceso productivo antes de que la producción final se recupere. Asimismo, debido a que la curva de la demanda (D) deriva de la curva de transformación $\left(P_{p}\right)$ y $\mathrm{D}$ disminuye más lentamente que la producción final $\left(P_{T}\right)$, la producción en proceso $\left(P_{p}\right)$ debe hacer lo mismo. Por ese motivo, en el gráfico $2, P_{p}$ disminuye a un ritmo menor que $P_{T}$.
}

\section{VII}

\section{De la economía cerrada a la dinámica} centro-periferia

Prebisch utiliza el marco anterior para analizar la relación entre el centro y la periferia. Ya había empleado ampliamente esta dicotomía analítica cuando, en sus primeros análisis de la economía argentina, se dio cuenta de que el ciclo formaba parte de un proceso global que comprendía un centro cíclico y una periferia ${ }^{8}$.

\footnotetext{
${ }^{8}$ Véanse Prebisch (2003c y 1993b), y Pérez Caldentey y Vernengo (2011).
}

En la teoría dinámica centro-periferia se asume que la periferia se especializa totalmente en la producción de materias primas, que se exportan al centro a cambio de manufacturas. Los beneficios de los sectores final, minorista y mayorista se obtienen y gastan en el centro. Los beneficios de la producción de materias primas se obtienen en la periferia y se gastan en el centro (es decir que se transfieren al centro). En consecuencia, las empresas del centro atienden a una demanda que se 
origina tanto en el centro como en la periferia. Se asume además que el centro emite la moneda de reserva, que es la única moneda en la periferia. En otras palabras, la periferia se "dolariza". Si bien este supuesto puede parecer extremo, refleja el hecho de que las importaciones de los países periféricos deban pagarse en la moneda clave de un país central. Mientras la periferia no tenga autonomía en materia de políticas, desempeñará un papel pasivo y constituirá el espacio de circulación de los ingresos enviados desde el centro (Prebisch, 1993h). De acuerdo con Prebisch, el supuesto de pasividad por parte de la periferia es realístico.

Prebisch aplica al centro y a la periferia su análisis dinámico de una economía cerrada, según el cual la demanda tiende a superar a la oferta en la fase ascendente del ciclo debido a la diferencia entre el tiempo necesario para que los ingresos regresen a las empresas (como demanda) y el tiempo necesario para terminar la producción (oferta). Esta diferencia provoca un incremento del precio y de los beneficios, que a su vez justifica compromisos de producción adicionales.

La aplicación de este marco a un modelo centroperiferia supone que la realización del proceso productivo por el centro necesariamente produce una demanda neta positiva (una inyección) en el centro. De ese modo, el centro registra un exceso de demanda. Sin embargo, también registra al mismo tiempo salidas equivalentes al valor de las importaciones de materias primas de la periferia. Esto constituye el flujo de ingresos pagados a las empresas y los trabajadores de la periferia por la producción y la exportación de las materias primas vendidas al centro. A su vez, las empresas y los trabajadores de la periferia compran los productos finales fabricados en el centro. De ahí que el flujo de ingresos gastados en la periferia por las empresas del centro regresa al centro.

Por una parte, Prebisch postula - como en su análisis anterior - que el tiempo necesario para que los ingresos vuelvan a las empresas es más breve que el período requerido para llevar los productos terminados al mercado. Y por otra, que el tiempo necesario para que los ingresos regresen de la periferia al centro (demanda de la periferia) es mayor que el tiempo necesario para que la producción final del centro se lleve al mercado y se venda (en el centro) (Prebisch, 1993f). Como resultado, se observan al mismo tiempo un exceso de demanda de origen céntrico y una insuficiencia de demanda de productos finales de origen periférico.

La interacción entre las fuerzas y los factores que determinan el exceso de demanda en el centro y la insuficiencia de demanda en la periferia constituye el núcleo de la dinámica de Prebisch aplicada a la dicotomía centro-periferia. Al comienzo de la fase ascendente, la demanda excede a la oferta y conduce a un incremento de los precios y los beneficios. Simultáneamente, una parte de los mayores ingresos sale hacia la periferia, controlando la presión sobre la demanda agregada neta. Cuanto mayor sea el coeficiente de salida, menor será el exceso de demanda neta y, por ende, menores los beneficios y los incentivos para la expansión constante en el centro.

Prebisch sostiene que el exceso neto de demanda disminuirá con el tiempo debido al aumento de la elasticidad con respecto al ingreso de las importaciones de productos demandados de la periferia por el centro. El flujo de beneficios (del centro a la periferia y viceversa) actúa como la fuerza equilibradora entre la demanda y la oferta en el centro. En sus palabras:

“(...) hay un exceso neto de demanda sobre oferta en el centro cíclico, que tiene como consecuencia la disminución de las existencias de los empresarios finales, lo cual causa un aumento de demanda entre empresarios con el fin de aumentar la producción, que trae como consecuencia el aumento de beneficios el cual, en la hipótesis que estamos considerando, va en una mayor parte a parar a la periferia. Es decir que mientras no se llegue al punto de conjunción habrá una continua disminución de existencias, un continuo ascenso de la demanda entre empresarios, un continuo crecimiento de beneficios y un desplazamiento de beneficios hacia la periferia. Los beneficios se siguen desplazando de más en más a la periferia, hasta que su cuantía haya creado una insuficiencia igual al exceso. Ese es el juego espontáneo del sistema" (Prebisch, 1993f).

Este mecanismo se refuerza por el supuesto de que las empresas necesitan más tiempo para recuperar sus ganancias de la periferia que para llevar su producción final al mercado y venderla. Precisamente por esta razón, Prebisch argumenta que el punto de convergencia o equilibro entre el exceso de demanda en el centro y la insuficiencia de demanda en la periferia sería muy difícil de alcanzar, sobre todo cuando - como asumió en su modelo- el coeficiente de salida del centro es mucho mayor que el de la periferia. Proporciona un ejemplo específico en que el coeficiente de salida tiene un valor de 0,50 en el centro y 0,20 en la periferia (Prebisch, 1993g): "El coeficiente de salida, o sea el que nos da el retorno de ingresos, es, según lo que ya explicamos, mucho más alto en la masa de ingresos de origen céntrico, que en la masa de ingresos de origen periférico". 
En ese marco, Prebisch asume que los ingresos aumentarían 100 unidades constantes en el centro y solo 20 unidades en la periferia (véase el gráfico 3). El resultado es una brecha cada vez mayor entre la cantidad que el centro gasta en la periferia y la cantidad que la periferia gasta en el centro, indicada por la distancia entre la línea punteada y la línea negra (entre los puntos A y B). La línea punteada muestra la evolución de las salidas para el centro con sucesivos aumentos del ingreso de 100 unidades y un coeficiente de importaciones de 0,50. La línea negra traza las salidas para la periferia en el supuesto de sucesivos incrementos en el ingreso de 20 unidades y un coeficiente de importaciones de 0,20. Sin embargo, incluso si los ingresos en el centro y la periferia se incrementaran en la misma cantidad, por ejemplo 100 unidades, la brecha continuaría aumentando (aunque en menor medida). En el gráfico 3 esto se aprecia en la diferencia entre la línea punteada y las dos líneas debajo de ella, que trazan las salidas para la periferia en el supuesto de sucesivos incrementos del ingreso de 100 y 200 unidades, con un coeficiente de importaciones de 0,20 en ambos casos.

GRÁFICO 3

Coeficientes de salida (importaciones) en el centro y la periferia

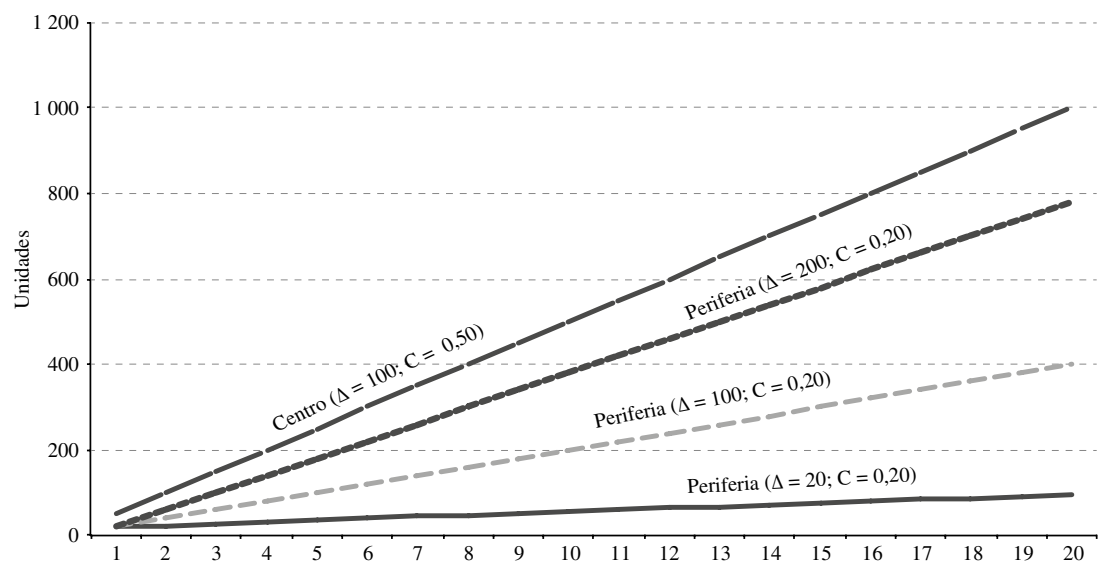

Fuente: Elaboración propia sobre la base de R. Prebisch, "Teoría dinámica de la economía (vi)", Raúl Prebisch: Obras, 1919-1948, vol. 4, Buenos Aires, Fundación Raúl Prebisch, 1993.

El hecho de que Prebisch asuma, por lo menos hasta su séptima conferencia sobre dinámica económica (Prebisch, 1993h), que el coeficiente de importaciones es mayor en el centro que en la periferia parece una total contradicción con su pensamiento. Un principio clave del trabajo de Prebisch, y de la escuela estructuralista, es que la elasticidad con respecto al ingreso de las importaciones en la periferia es mayor que en el centro (véase, por ejemplo, Prebisch, 1949). Desde el punto de vista adoptado en este trabajo, esta contradicción puede explicarse mediante dos hipótesis.

En primer lugar, Prebisch sabía que, desde alrededor de 1926, los países en desarrollo habían experimentado una caída de los términos de intercambio decreciente que constituye un efecto en los precios relativos. Sin embargo, no examinó específicamente las diferencias en los efectos ingreso entre el centro y la periferia, incluso cuando abordó el tema del comercio entre un país desarrollado y uno en desarrollo en una conferencia de 1948, titulada "El esquema clásico del comercio internacional y el oro en la realidad", como parte de su curso sobre dinámica económica ${ }^{9}$. Tal vez Prebisch comenzó a ser explícitamente consciente de la importancia de los efectos ingreso en el sector externo durante el período en que estaba desarrollando su teoría dinámica, pero en 1948 todavía no había incorporado completamente

\footnotetext{
9 Este texto aparece con el título "Progreso técnico y comercio internacional" en las obras completas de Prebisch (Prebisch, 1948g, págs. 363-374). Sin embargo, el autor era muy consciente de la dependencia de las importaciones de los países en desarrollo, como lo demuestra su análisis de los efectos de la Gran Depresión. Al concentrarse en los efectos ingreso después esta gran crisis, argumentó que las importaciones aumentan porque una parte considerable del consumo directo de un país, o la maquinaria y los materiales para sus industrias, provienen del exterior, y el incremento de los ingresos se refleja en mayores niveles de importaciones (Prebisch, 1991b).
} 
esos efectos en su modelo ${ }^{10}$. La segunda hipótesis, menos creíble, es que Prebisch utilizó el ejemplo de un coeficiente de importaciones mayor en el centro que en la periferia solo como recurso expositivo para facilitar la presentación de su teoría. Aun así, cuando Prebisch abandonó este supuesto no desarrolló en forma detallada el caso hipotético contrario (es decir, aquel en que el coeficiente de importaciones es mayor en la periferia que en el centro).

De acuerdo con el funcionamiento de la dinámica de Prebisch, la creciente brecha de gastos conduce eventualmente a una mayor redistribución de los beneficios hacia la periferia y, por consiguiente, a un incremento del ingreso en la periferia, que a su vez se traduce en una reducción de la brecha. En otras palabras, a partir de una situación en que los coeficientes de salida son 0,50 y 0,20 respectivamente en el centro y la periferia, con aumentos de ingresos de 100 y 20 unidades, como indican las líneas respectivas, la brecha comenzaría eventualmente a reducirse como consecuencia de la redistribución de los beneficios (llevando a un cambio de C a D en el gráfico 3).

Prebisch pensaba que este proceso dinámico conduciría a un mayor incremento de los beneficios e ingresos en la periferia que en el centro (Prebisch, 1993f). Esto, junto con una menor salida de los ingresos de la periferia hacia el centro (con respecto a la salida del centro a la periferia), eventualmente compensaría con creces el exceso neto de demanda en el centro, creando una situación de exceso neto de oferta. En la medida en que los fabricantes de productos finales se encontraran con un nivel de existencias mayor que el planeado, reducirían los pedidos y la demanda, lo que tendría un efecto cascada en la cadena de productores. La demanda, los precios y los beneficios más bajos causarían una recesión en el centro y la menor demanda del centro de productos de la periferia reduciría los beneficios y el nivel de actividad en la periferia.

En palabras de Prebisch:

"Cuando había un exceso neto de demanda disminuían las existencias de los empresarios y esto los llevaba a acrecentar la demanda entre empresarios primero, y luego a acrecentar la producción. Y

\footnotetext{
10 Véase la entrevista de Mallorquín a Ifigenia Martínez, quien trabajó con Prebisch entre 1949 y 1950. Ella menciona que cuando Prebisch visitó México en 1949, estaba muy preocupado por el sector externo y la tendencia al desequilibrio en ese sector a medida que un país se desarrolla y cambia su estructura económica. También sostiene que el modelo de desarrollo de la CEPAL, especialmente con respecto al sector externo, se basa en la experiencia mexicana (Mallorquín, 1998, pág. 147).
}

cuando hay una insuficiencia neta de demanda aumentan las existencias invendibles en manos de los empresarios, éstos se ven llevados a contraer la producción. Si admitimos esta relación entre la variación de las existencias y la conducta de los empresarios, no es concebible que los empresarios sigan pagando a los factores productivos del centro y comprando en la periferia las mismas cantidades, cuando la producción se va acumulando sin venderse (...)" (Prebisch, 1993g, pág. 469).

La relación entre las existencias y la demanda es un eslabón decisivo en el mecanismo de transmisión y un elemento fundamental de la dinámica de Prebisch y de sus argumentos contra "la economía del equilibrio". El autor lo explica de la siguiente manera: "Si pudiéramos eliminar totalmente la acción de las existencias sobre la demanda, llegaríamos a la posición de equilibrio. Pero ello sería eliminar el único medio de acción que tiene el empresario en el régimen capitalista: sería sustraer al sistema una de sus partes vitales. Mientras esa parte vital exista y las reacciones de los empresarios - no todasse produzcan, el sistema no alcanzará el equilibrio". El aumento (la reducción) de las existencias refleja, a su vez, la importancia de las expectativas como factor determinante de las decisiones de incrementar (aminorar) la producción. En la fase ascendente, cuando debido a la mayor demanda "los empresarios ven disminuir sus existencias, no solamente se preocupan de elevar la producción para atender el nuevo nivel que calculan en la demanda, sino también se anticipan a posibles ritmos de crecimiento de acuerdo con las circunstancias del Mercado (...) Lo mismo sucede en el descenso" (Prebisch, 1993g).

De acuerdo con Prebisch, su teoría era de carácter general y no se limitaba a casos y supuestos específicos. En consecuencia, hizo tres modificaciones a su modelo: introdujo el capital fijo, tuvo en cuenta una expansión autónoma de la demanda en la periferia mediante la creación de crédito y consideró la posibilidad de un coeficiente de importaciones más alto en la periferia que en el centro.

El uso de bienes de capital circulante y fijo en la elaboración de productos finales determina que el proceso productivo sea más largo de lo que sería si solo se utilizaran bienes de capital circulante. En su modelo, Prebsich asume que la creación de crédito ("ahorro forzado") financia la producción de capital circulante y que el "ahorro voluntario", en forma de fondos acumulados durante un período de contracción, financia la inversión en bienes de capital fijo. De ahí que la introducción de la inversión en capital fijo en el modelo de Prebisch agrega 
una fuente adicional de demanda a la originada en la inversión en capital circulante. Los ingresos y beneficios pagados en el curso de la producción de capital fijo se suman a los distribuidos en el proceso de producción de capital circulante (Prebisch, 1993e). La fuente adicional de demanda, ingresos y beneficios se atenuará por la cantidad de salida a la periferia. Como Prebisch afirma:

"Pero las inversiones se hacen también tomando de la periferia materias primas y artículos en proceso. De manera que solamente una parte de los beneficios del centro cíclico se van a transformar en ingresos de los factores productivos del centro: la otra parte se va a transformar en pagos de artículos en proceso a los empresarios de la periferia. Es decir que al utilizarse los beneficios en inversiones fijas, una parte de esos beneficios va a salir inmediatamente a la periferia y quedará sujeta, por lo tanto, al ritmo de los retornos de la periferia" (Prebisch, 1993h). Prebisch asume que la inversión total precede inicialmente a los beneficios, hasta que los empresarios terminan los ahorros utilizados para financiar bienes de capital. De ahí en adelante, las empresas solo invierten en bienes de capital circulante, utilizando el ahorro y la creación de crédito para financiar nuevas inversiones, y los beneficios superan la inversión. El "exceso de beneficios" puede consumirse en forma de productos finales o ahorrarse y reinvertirse para incrementar el capital circulante. Cuanto más se ahorre y reinvierta el exceso de beneficios, menos dependerán las empresas de la creación de crédito. Debido a que algunos beneficios se gastan en la periferia, disminuye

\section{VIII}

\section{Conclusión}

El largo proceso de desarrollo de las ideas económicas de Prebisch comenzó en la década de 1920 y continuó a fines de la década de 1940 con su teoría dinámica de la economía. La esencia del análisis dinámico de Prebisch, en que el ciclo y el crecimiento van de la mano, es la introducción de la diferencia entre el tiempo requerido para la circulación de los ingresos en el proceso productivo y el tiempo necesario para la colocación y venta de la producción final en el mercado. En tal sentido, forma parte de una gran tradición de autores que intentaron formalizar la macrodinámica tras la revolución keynesiana. Prebisch mantiene elementos de espíritu keynesiano la demanda de productos finales. Sin embargo, a través del mecanismo descrito anteriormente, el punto de convergencia entre el exceso de demanda de origen céntrico y la insuficiencia de demanda se alcanzará con el tiempo. La introducción del capital fijo no modifica las premisas básicas del análisis de Prebisch, sino que simplemente introduce una desviación en el camino hacia el punto de convergencia.

La segunda modificación introducida por Prebisch supone que la periferia no juega un papel pasivo y no depende completamente de la moneda del centro, sino que en cambio depende en cierta medida de su propio aumento de la creación de crédito para financiar sus actividades de producción. En este marco hipotético, a diferencia de aquel en que la periferia solo desempeña un papel pasivo, la demanda será mayor tanto en el centro como en la periferia. Sin embargo, Prebisch no desarrolló esta hipótesis en forma detallada. Más que una hipótesis con aplicación práctica o que reflejaba el desarrollo capitalista, la consideraba una curiosidad (Prebisch, 1993i).

La última modificación, en que el coeficiente de importaciones de la periferia es mayor que el del centro, simplemente disminuye el tiempo necesario para alcanzar el punto de convergencia. Prebisch no consideró que este fuera un supuesto importante, incluso aunque desempeñó un papel fundamental en la lógica de su obra El desarrollo económico de la América Latina y algunos de sus principales problemas, que publicó algunos meses después de su última conferencia sobre dinámica económica. junto con otros decididamente neoclásicos, en tanto que al mismo tiempo introduce elementos de la antigua escuela clásica, como era de esperarse en un período de transición de la profesión económica. Como también se podía esperar de un autor que creció en un ambiente bastante ecléctico desde el punto de vista intelectual.

No obstante, Prebisch se destaca entre sus contemporáneos por su intento de explicar el crecimiento cíclico de la economía global como el resultado de la interacción entre el centro y la periferia, en que la división internacional del trabajo es importante. No solo examina la especificidad de los problemas de 
administrar la economía periférica, sino que es el único de los economistas que se ocupó del crecimiento cíclico que señala la importancia del desplazamiento del centro global del Reino Unido de Gran Bretaña e Irlanda del Norte a los Estados Unidos de América durante el período de entreguerras.

Con el tiempo, su concepción de la especificidad institucional e histórica de la dinámica económica evolucionaría hasta convertirse en lo que los estructuralistas de la CEPAL denominaron método de análisis histórico- estructural, que examinaba el proceso de transformación estructural de las economías en desarrollo desde una perspectiva histórica. La comprensión de la dinámica capitalista de Prebisch, antes de escribir su Manifiesto y convertirse en Secretario Ejecutivo de la CEPAL, se basaba en una teoría que debía ser general y universal —ir mucho más allá de los problemas de los países periféricos con relaciones de intercambio decrecientesy que se convirtió en el sello distintivo de sus aportes al análisis económico.

\section{Bibliografía}

CEPAL (Comisión Económica para América Latina y el Caribe) (1951), Estudio Económico de América Latina (E/CN.12/164/ Rev.1), Nueva York, Naciones Unidas.

Kalecki, M. (1971), Selected Essays on the Dynamics of the Capitalist Economy, 1933-1970, Cambridge, Cambridge University Press.

Keynes, J.M. (1943), Teoría general de la ocupación, el interés y el dinero, Ciudad de México, Fondo de Cultura Económica. Publicado originalmente en 1936.

Mallorquín, C. (1998), Ideas e historia en torno al pensamiento económico latinoamericano, Ciudad de México, Plaza y Valdés.

Pérez Caldentey, E. y M. Vernengo (2011), "Retrato de un joven economista: la evolución de las opiniones de Raúl Prebisch sobre el ciclo económico y el dinero, 1919-1949", Revista CEPAL, $\mathrm{N}^{\circ} 106$ (LC/G.2518-P), Santiago, Comisión Económica para América Latina y el Caribe (CEPAL).

Prebisch, R. (2003a), "Introducción a la teoría dinámica de la economía", Dr Raúl Prebisch, 1901-1986: archivo de trabajo, J.F. Besa García (ed.), Santiago, Microfilmación y Sistemas Microsystem, S.A.

(2003b), "La teoría keynesiana y la realidad cíclica", $D r$. Raúl Prebisch, 1901-1986: archivo de trabajo, J.F. Besa García (ed.), Santiago, Microfilmación y Sistemas Microsystem, S.A. (2003c), "La crisis de la economía política y la revolución keynesiana", Dr. Raúl Prebisch, 1901-1986: archivo de trabajo, J.F. Besa García (ed.), Santiago, Microfilmación y Sistemas Microsystem, S.A.

(1993a), Raúl Prebisch: Obras 1919-1949, vol. 4, M. Fernández López (ed.), Buenos Aires, Fundación Raúl Prebisch.

(1993b), "Teoría dinámica de la economía (I)", Raúl Prebisch: Obras, 1919-1948, vol. 4, Buenos Aires, Fundación Raúl Prebisch.

(1993c), "Teoría dinámica de la economía (II)", Raúl Prebisch: Obras, 1919-1948, vol. 4, Buenos Aires, Fundación Raúl Prebisch.

(1993d), "Teoría dinámica de la economía (III)", Raúl Prebisch: Obras, 1919-1948, vol. 4, Buenos Aires, Fundación Raúl Prebisch.

(1993e), "Teoría dinámica de la economía (Iv)", Raúl Prebisch: Obras, 1919-1948, vol. 4, Buenos Aires, Fundación Raúl Prebisch.

(1993f), "Teoría dinámica de la economía (v)", Raúl Prebisch: Obras, 1919-1948, vol. 4, Buenos Aires, Fundación Raúl Prebisch.

(1993g), "Teoría dinámica de la economía (vi)", Raúl Prebisch: Obras, 1919-1948, vol. 4, Buenos Aires, Fundación Raúl Prebisch.

(1993h), "Teoría dinámica de la economía (VII)", Raúl Prebisch: Obras, 1919-1948, vol. 4, Buenos Aires, Fundación Raúl Prebisch. (1993i), "Teoría dinámica de la economía (VIII)", Raúl Prebisch: Obras, 1919-1948, vol. 4, Buenos Aires, Fundación Raúl Prebisch.

(1993j), "Panorama general de los problemas de regulación monetaria y crediticia en el continente americano: América Latina", Raúl Prebisch: Obras, 1919-1948, vol. 4, Buenos Aires, Fundación Raúl Prebisch.

(1993k), "Los planes monetarios internacionales, la moneda y los ciclos económicos en la Argentina", Raúl Prebisch: Obras, 1919-1948, vol. 4, Buenos Aires, Fundación Raúl Prebisch.

(19931), "La experiencia monetaria argentina desde la crisis mundial y la creación y funcionamiento del banco central, la moneda y los ciclos económicos en la Argentina", Raúl Prebisch: Obras, 1919-1948, vol. 4, Buenos Aires, Fundación Raúl Prebisch.

(1991a), Raúl Prebisch: Obras 1919-1948, vols. 1, 2 y 3, M. Fernández López (ed.), Buenos Aires, Fundación Raúl Prebisch.

(1991b), "Tendencias del balance de pagos", Raúl Prebisch: Obras 1919-1948, M. Fernández López (ed.), Buenos Aires, Fundación Raúl Prebisch.

(1951a), "Interpretação do processo de desenvolvimiento econômico", Revista Brasilera de Economía, vol. 5, ํ 1 .

(1951b), "Crecimiento, desequilibrio y disparidades: interpretación del proceso del desarrollo económico", Estudio económico de América Latina (E/CN.12/164/Rev.1), Nueva York, Naciones Unidas.

(1949), El desarrollo económico de la América Latina y algunos de sus principales problemas (E/CN.12/89), Santiago, Comisión Económica para América Latina y el Caribe (CEPAL). (1948a), "Introducción al curso de dinámica económica", Revista de la Facultad de Ciencias Económicas, vol. 1, $\mathrm{N}^{\circ} 4$, Buenos Aires, Universidad de Buenos Aires.

(1948b), "La tasa de interés en las doctrinas clásica y keynesiana”, Apuntes de Economía Política, Buenos Aires.

(1948c), "La tasa de interés como reguladora del sistema económico", Apuntes de Economía Política, vol. 4, Buenos Aires.

(1948d), "Los tres planos independientes de la teoría clásica", Apuntes de Economía Política, Buenos Aires.

(1948e), "Papel del beneficio en la realidad capitalista", Apuntes de Economía Política, Buenos Aires.

(1948f), "Cotejo entre el esquema clásico y la realidad cíclica", Apuntes de Economía Política, Buenos Aires.

(1948g), "Progreso técnico y comercio internacional", Apuntes de Economía Política, Buenos Aires.

(1948h), Apuntes de Economía Política, Buenos Aires.

(1947a), Introducción a Keynes, Buenos Aires, Fondo de Cultura Económica. 
(1947b), "Programa de economía política (dinámica económica)", Buenos Aires, Universidad de Buenos Aires. (1984), "Cinco etapas en mi pensamiento sobre el desarrollo", Pioneros del desarrollo, G. Meier y D. Seers (eds.), Madrid, Editorial Tecnos.

(1981), Capitalismo periférico: crisis y transformación, Ciudad de México, Fondo de Cultura Económica.
Schumpeter, J. (1946), "John Maynard Keynes 1883-1946”, American Economic Review, vol. 36, $\mathrm{N}^{\circ} 4$, Nashville, Tennessee, American Economic Association.

Termini, V.A. (1981), "Logical, mechanical and historical time in economics", Economic Notes, vol. 10, $\mathrm{N}^{\circ} 3$, Monte dei Paschi di Siena. 